\title{
Direct Observational Evidence for Magnetic Fields in Hot Stars
}

\author{
Gautier Mathys \\ European Southern Observatory, Casilla 19001, Santiago 19, Chile
}

\begin{abstract}
Attempts at achieving direct detections of magnetic fields in hot stars are reviewed. The techniques used in these observations and their analysis are described, with emphasis on the physical situations to which they are relevant. Results of projects carried out in the last couple of years are reported, and prospects for future investigations are briefly considered.
\end{abstract}

\section{Introduction}

As emphasized in many contributions at this meeting, an increasing number of observations of hot stars provide indirect evidence that magnetic fields must be present in those stars. Such observations include, for instance, rotationally modulated winds in $\mathrm{O}$ stars, X-ray emission in Be stars, and transient features in the profiles of absorption lines in the visible spectrum of Be stars. This has triggered, in the last few years, an intensification of the efforts towards direct detections and measurements of magnetic fields in those stars. In this contribution, I review the current status of our knowledge in this area.

\section{Diagnosis Methods and Brief History}

Stellar magnetic fields are accessible to direct observation through the Zeeman effect that they induce in spectral lines. The most straightforward manifestation of this effect is the splitting of the lines into several components. The wavelength separation of adjacent split components depends on the atomic properties of the levels involved in the considered transition, but typically is of the order of $4.6710^{-13} \lambda^{2}\langle H\rangle$, where $\lambda$ is the nominal wavelength of the transition (expressed in $\AA$ ) and $\langle H\rangle$ is the mean magnetic field modulus (in $\mathrm{G}$ ), that is, the line-intensity weighted average over the visible stellar hemisphere of the modulus of the magnetic vector. Hence for a line at, say, $5000 \AA$, the separation of magnetically split components per $\mathrm{kG}$ of field strength is of the order of $10^{-2} \AA$ or, in velocity units, $0.6 \mathrm{~km} \mathrm{~s}^{-1}$. Not only can this effect be observed only in spectra recorded at sufficiently high resolution of stars with fairly considerable and uniform ${ }^{1}$ magnetic fields, but also it

\footnotetext{
${ }^{1}$ If there is a wide dispersion of field strengths over the stellar surface, the contributions to the observed line coming from different parts of the stellar surface
} 
may easily be smeared out through broadening of the magnetically split line components by other effects such as, primarily, Doppler effect due to stellar rotation and/or local atmospheric motions. In the kind of stars considered in this meeting, such motions are often quite significant and ruin virtually every chance of direct observation of magnetic line splitting.

In principle, even when magnetic line splitting cannot be directly observed, it remains possible to detect stellar magnetic fields from observations of the differential broadening effect that they induce in lines of different magnetic sensitivity. This approach has been successfully applied to a number of stars, mostly either active late-type stars or magnetic Ap and Bp stars. But again, its suitability for the study of the stars of interest at this colloquium is limited. Indeed, although it should in principle be possible to diagnose the magnetic field from only two lines of very different magnetic sensitivity, in practice, our ability to do this is limited by the uncertainties introduced by possibly unrecognized effects that may alter the shapes of either of the two lines and may be misinterpreted as due to a magnetic field. This possibility appears especially critical for hot stars with extended atmospheres, where different lines are often formed at different depths, possibly in different velocity fields, with different departures from LTE, etc. The usual workaround is to use a larger number of lines (ideally, a statistically significant sample) for the differential magnetic broadening study. This workaround is bound to be much less successful for hot stars than for solar-type or Ap stars, the spectra of which contain an incomparably larger number of lines suitable to magnetic field diagnosis.

Therefore, the best chances for a successful diagnosis of the magnetic fields of hot stars rest on the exploitation of the polarization induced in the spectral lines by the Zeeman effect. To first order, the result of the latter is a shift in the wavelength of the centre of gravity of a line between observations performed in right and left circular polarizations (RCP and LCP, resp.). The order of magnitude of this shift is $9.3410^{-13} \lambda^{2}\left\langle H_{z}\right\rangle$, which at first glance is similar to the separation of adjacent components of resolved lines. However, there are two differences. First, rather than splitting within a line, the effect is, in the present case, the global shift of a whole line. The latter is much easier to detect than the former, because in particular other effects affecting the line shape are essentially similar in both circular polarizations, hence do not mask the magnetically induced differences between the latter. Yet, while the magnetic splitting is proportional to the mean field modulus $\langle H\rangle$, the observed wavelength shift between RCP and LCP is proportional to the mean longitudinal field $\left\langle H_{z}\right\rangle$. The latter is the line-intensity weighted average over the visible stellar disk of the component of the magnetic vector along the line of sight. Due to its vectorial nature, this average will differ from zero

will be characterized by different line splittings, which once integrated over the visible stellar disk may broaden the split line components to such an extent that they are no longer resolved. 
only provided that the star's magnetic field has a suitable large-scale organization: for complex field structures (e.g., such as in the sun), where the star is covered by a large number of small regions where fields of opposite polarities but similar strengths prevail, the contributions of these small regions to the disk-integrated spectral lines average out to a large extent, so that the lines show no net wavelength shift between RCP and LCP. Admittedly, in some circumstances (thanks, e.g., to different rotational Doppler shifts of regions of different field polarities), higher-order effects may still detectable as differences between the line profiles recorded in RCP and LCP: this possibility, which anyway involves significant additional complexity, will be briefly considered later in this contribution.

The observation of global wavelength shifts between spectral lines recorded in RCP and LCP (and the related applications, such as Balmer line photopolarimetry: see Landstreet 1982) has been so far the main source of stellar magnetic field measurements. The vast majority of the stars in which definitive magnetic field detections have been achieved through this technique are Ap and Bp stars. These stars have magnetic fields which, in first approximation, are nearly dipolar; the dipole axis is generally inclined at some non-zero angle with respect to the stellar rotation axis. This simple dipolar field structure is a very favourable one for the observation of a global wavelength shift of disk-integrated spectral lines between RCP and LCP.

The lower temperature limit of the magnetic Ap star phenomenon lies around spectral type F0 and is understood to result from the onset in cooler stars of significant convection in outer layers. There is no such discontinuity in the stellar physical properties towards the high temperatures: therefore it appears rather natural, in a first step, to try to detect magnetic fields in hot stars through the techniques that have been successfully applied for Ap and Bp stars. In particular, the hottest magnetic Bp stars currently known are of spectral type $\mathrm{B} 1$, and there is no reason to believe a priori that large-scale organized magnetic fields and/or chemical photospheric peculiarities should not exist in higher-temperature stars. It has, more plausibly, been argued that there must be less detections of such phenomena in the hottest B stars and in $O$ stars than in lower-temperature stars as a result of the combination of the smaller total number of $O B$ stars and of the increased difficulty to detect fields and spectral anomalies in them, due to the specific characteristics of their spectra (low density of spectral lines, broadened and/or distorted by various dynamical effects). Accordingly, all the early attempts at detecting magnetic fields in hot stars have aimed at determining their mean longitudinal magnetic field from the wavelength shifts of their spectral lines between opposite circular polarizations. H.W. Babcock had investigated approximately a dozen $\mathrm{Oe} / \mathrm{Be}$ stars in his seminal survey of stars in search of magnetic fields (Babcock 1958): two of them only showed fairly doubtful hints of the presence of a magnetic field. Among the various attempts made since Babcock's work until the last couple of years, some of the best ones (with measurement uncertain- 
ties of the order of $100 \mathrm{G}$ or less) include the work of Barker et al. (1981) on the O4ef star $\zeta$ Pup, Landstreet's (1982) investigation of a sample of apparently normal upper main-sequence stars, and the study of a sample of $\mathrm{Be}$ stars by Barker et al. (1985). Neither of these (nor any of the contemporary works not explicitly listed here) yielded any definite field detection.

\section{Recent Searches for Magnetic Fields in Hot Stars}

Not only do the attempts at magnetic field detections in hot stars described in the previous section all aim at exploiting the same manifestation of the Zeeman effect, but also they all were made using basically only two types of instruments. One is the original Babcock-type photographic Zeeman analyzer, through the use of which two high-dispersion stellar spectra corresponding to incoming light of opposite circular polarizations are simultaneously recorded next to each other on a photographic plate. The other is the Balmer line photopolarimeter, which transmits alternatively to a photomultiplier tube RCP and LCP light issued from a narrow region of the wing of a Balmer line of hydrogen, isolated by use of an interference filter. The difference between the signals corresponding to incoming RCP and LCP is computed on the fly by the electronics associated with the photomultiplier tube, which is equivalent to measuring the wavelength shift of the line between the two polarizations. While the Babcock-type analyzer suffers from the inherent limitations of the photographic plate in terms of noise, the achievable ratio between polarimetric signal and noise in Balmer line photopolarimetry is limited by the smallness of the former, as a result of the fact that Balmer lines are very broad in early-type stars.

In recent years, however, a number of new instruments have entered into operation, through which high resolution spectra are recorded simultaneously in two mutually orthogonal (circular or linear) polarizations on a CCD, hence combining the strengths of the Babcock-type and Balmer line analyzers and overcoming their limitations to a large extent. Several of these new instruments have been used by various groups for studies of magnetic fields of hot stars: the Zeeman analyzer of the ESO CASPEC spectrograph (described by Mathys and Hubrig 1997), the polarimeter built in Meudon for use on various spectrographs (Donati et al. 1997), the MuSiCoS spectropolarimeter at the Pic-du-Midi Observatory (Donati et al. 1998), and the William-Wehlau spectropolarimeter (Eversberg et al. 1998).

In parallel, new methods have been developed for the analysis of the data, which allow one not only to interpret the global wavelength shift of lines between RCP and LCP, but also to exploit the additional information that may exist in differences between the shapes of the lines in the two polarizations. These techniques have already proved successful in works on other types of stars. The Zeeman-Doppler imaging (Semel 1989) has allowed Donati et al. (1990) to achieve the first spectropolarimetric determinations of magnetic 
fields in active late-type stars. The moment technique (Mathys 1988) has proved quite effective in providing new constraints on the geometrical structure of the magnetic fields of Ap and Bp stars (Mathys 1993). A first glimpse of the large potential of the least-square deconvolution method has been given by Donati et al. (1997). On the basis of these positive results, these new techniques appear very promising for studies of hot star magnetic fields. They only start to be applied in such studies, but this is undoubtedly one of the ways to go for the future. Yet, until now, most of the results obtained with the new generation instruments have followed from the classical approach of measuring the wavelength shifts of lines between RCP and LCP. These recent results are briefly reviewed below.

The hot star for which the largest number of attempts at detecting a magnetic field have been made in the last few years is definitely the O7V star $\theta^{1}$ Ori C. The interest for this star has been triggered by the reports by the Heidelberg group that it shows a phenomenology very similar to that of magnetic oblique rotators of the Ap and $\mathrm{Bp}$ group, such as, in particular, periodic variations of line intensities. This raised high hopes that $\theta^{1}$ Ori $\mathrm{C}$ might be the first representative of the long sought after hot extension of the magnetic chemically peculiar A and B stars into the $\mathrm{O}$ spectral type class. Should this be the case, the star would logically be expected to harbour a strong, roughly dipolar magnetic field. However, the attempts made by Donati and Wade (1998) with MuSiCoS, Eversberg et al. (1998) with the William-Wehlau polarimeter, and myself with various collaborators using CASPEC (Mathys et al., in preparation), all yielded null measurements of the mean longitudinal field, with typical uncertainties of the order of $250 \mathrm{G}$. Donati and Wade compute that, for a dipolar field geometry, this corresponds to an upper limit of the polar field strength of $1.6 \mathrm{kG}$. Incidentally, these authors also detected a strong circular polarization of the continuum, which definitely does not arise from a magnetic field but whose origin remains for the time being an enigma. Another promising candidate, the 07.5 giant $\xi$ Per, whose wind undergoes cyclical variability, has been observed at the CFHT through Balmer line photopolarimetry by Henrichs et al. (1998), who failed to detect a longitudinal magnetic field with $1 \sigma$ error bars of the order of $70 \mathrm{G}$.

Lex Kaper and myself conducted a search for magnetic fields in a sample of 10 fairly slowly rotating $O$ stars of various types, using CASPEC spectra. We determined the longitudinal fields with, in the best cases (half of the sample) $1 \sigma$ uncertainties of the order of $250-300 \mathrm{G}$, but we failed to achieve any definite detection. Some marginal measurements (between 2 and $3 \sigma$ ) suggest that weak fields might nevertheless possibly be present, which could become detectable through a fairly modest improvement (say, a factor of 2) of the measurement accuracies. A more detailed account of this work will be given in a forthcoming paper.

The above-described investigations of $\mathrm{O}$ stars were aimed at detecting global fields with sufficient organization at the scale of the whole star: in that 
respect, they were quite similar to the older studies such as those mentioned in Sect. 2. In such a study based on Balmer line photopolarimetry, Barker et al. (1985) had derived an upper limit of $250 \mathrm{G}$ for the longitudinal field of the Be star $\lambda$ Eri. However, these observations hardly constrain the possible existence of localized magnetic fields, which are often assumed to be responsible for the occurrence of transient phenomena in the spectrum of this star, such as ephemeral "dimples" or weak emissions in the line He I $\lambda 6678$ and in other He I lines. If this interpretation is correct, detection of such localized fields should in principle be achievable by observations of the line transients that they trigger, since the latter should indeed arise from the location of the confined magnetic spot, without dilution from the rest of the stellar disk. An attempt to apply this approach, made by Myron Smith and myself with CASPEC, was severely hampered by bad weather conditions, and the small number of observations that could actually be performed did not allow us to follow any transient from its appearance to its disappearance. However, some transients were observed for part of their existence. The analysis of these data is still in a rather preliminary stage. So far no clear evidence of detection of a magnetic field has been obtained, but final, more quantitative conclusions require further analysis work.

A definite detection in a class of stars not previously known to harbour magnetic fields has been achieved by Donati (1998), who applied the leastsquare deconvolution technique to measure a longitudinal field of the order of $50 \mathrm{G}$ in the Herbig Ae star HD 104237, thereby confirming the reality of a marginal detection achieved earlier (Donati et al. 1997). However, Herbig Ae stars are admittedly somewhat cooler than the vast majority of the stars of interest at this meeting.

\section{Prospects and Suggestions for Future Work}

The almost entirely negative outcome of the attempts made so far to detect magnetic fields in hot stars with non-axisymmetric and/or variable winds may seem discouraging. Should one jump to the conclusion that these stars do not have magnetic fields and give up all attempts to detect the latter? In my opinion, this would be premature: there are a number of reasons to believe that magnetic fields may indeed be present in hot stars although they have so far escaped detection.

On the one hand, magnetic fields have definitely been observed in a number of helium peculiar stars between spectral types B1 and B6. These stars do have trapped circumstellar plasmas and non-asymmetric winds governed by their magnetic fields (Shore 1998 and references therein). Their mass-loss rate may be lower than in the most extreme objects discussed in this colloquium, but they certainly have much in common with the latter in terms of underlying physics. Further interest arises from the fact that some at least of these stars may not be just oblique magnetic rotators, as widely accepted 
in the classical view that their group constitutes a hot extension of that of the magnetic Ap stars, but that instead they may be the first examples of an entirely new class of magnetic stars, in which the observed magnetic variations are due to stellar pulsation and not the result of the changing aspect of the visible stellar hemisphere as a result of stellar rotation. Arguments supporting this view come from the fact that the magnetic variations of the B2p He strong star HD 96446 are inconsistent with the oblique rotator model (Mathys 1994).

On the other hand, the upper limits derived so far for the magnetic fields of hot stars are not yet low enough to be inconsistent with the magnetic field intensities advocated to explain various observational manifestations taken as indirect evidence of the presence of fields. Achievement of better sensitivity in searches for magnetic fields may well allow detections to be finally made. While for cooler stars, such improvement has often been achieved by the simultaneous consideration of more spectral lines, in the present case, it should rather come from higher signal-to-noise single line observations. Potential progress may also come from detailed studies of the polarized line profiles, in the eventuality that the fields of hot stars have structures significantly more complex than those of the magnetic Ap and Bp stars. In summary, there remain a wealth of directions to be explored, and whether they eventually lead to magnetic field detections or not, they will without doubt yield new and deeper insight into the physics of the considered stars.

\section{References}

Babcock H.W., 1958, ApJS 3, 141

Barker P.K., Landstreet J.D., Marlborough J.M., Thompson I., Maza J., 1981, ApJ 250, 300

Barker P.K., Landstreet J.D., Marlborough J.M., Thompson I.B., 1985, ApJ 288, 741

Donati J.-F., 1998, private communication

Donati J.-F., Catala C., Wade G.A., et al., 1998, A\&A (in press)

Donati J.-F., Semel M., Carter B.D., Rees D.E., Cameron A.C., 1997, MNRAS 291, 658

Donati J.-F., Semel M., Rees D.E., Taylor K., Robinson R.D., 1990, A\&A 232, L1

Donati J.-F., Wade G.A., 1998, A\&A (in press)

Eversberg T., Moffat A.F.J., Debruyne M., et al., 1998, these proceedings

Henrichs H.F., de Jong J.A., Nichols J.S., et al., 1998, preprint

Landstreet J.D., 1982, ApJ 258, 639

Mathys G., 1988, A\&A 189, 179

Mathys G., 1993, in IAU Coll. No. 138, ASP Conf. Series vol. 44, p. 232

Mathys G., 1994, in IAU Symp. No. 162, Kluwer, Dordrecht, p. 169

Mathys G., Hubrig S., 997, A\&AS 124, 475

Semel M., 1989, A\&A 225, 456

Shore S.N., 1998, these proceedings 


\section{Discussion}

T. Eversberg: The Landstreet equation is used for absorption-line stars. Is it reasonable to apply this formalism to emission lines coming from expanding winds?

G. Mathys: Yes, polarisation effects due to the magnetic field are similar in emission or in absorption. However, emission likely originates from higher layers than absorption. Due to magnetic flux conservation, magnetic fields in regions where emission lines are formed should be weaker than in the regions of formation of absorption lines: accordingly, it is probably even more difficult to detect a magnetic field in emission lines than in absorption lines.

C. Rodrigues: Why couldn't cyclotron emission explain the high circular polarisation of $\theta^{1}$ Ori C?

G. Mathys: To achieve the observed level of continuum circular polarisation by a cyclotron effect, the magnetic field would be so strong that the lines would be badly distorted: this is not observed.

P. Stee: Maybe the proper technique will be to observe with the VLTI in polarised light, because the global polarisation can be zero whereas locally you can have a polarisation of $100 \%$.

G. Mathys: I agree.

A. Moffat: What is the typical S/N you have in your spectra to search for circular polarisation? Why not go for several 1000 ?

G. Mathys: The spectra that I discussed have $\mathrm{S} / \mathrm{N}$ ratios of $\sim 400$. It is limited by the instrumental configuration used, in particular the inter-order scattered light of the non-dispersed echelle format. For hot stars, the broad wavelength coverage provided by this format is not a strong asset, since only very few diagnostic lines are available in any case. Accordingly, it might be preferable to observe a single line or a very limited number of lines in a restricted wavelength range with a standard spectrograph. The instrumental signature in the latter can probably be handled better, which would allow one to achieve higher (very high) $\mathrm{S} / \mathrm{N}$, which of course is desirable. 